\title{
Structurally selective assembly of a specific macrobicycle from a dynamic library of pseudopeptidic disulfides.
}

\author{
María Lafuente, Ignacio Alfonso* and Jordi Solà*
}

\begin{abstract}
Molecular recognition is essential in many chemical and biological processes. Studying the behavior of pseudopeptides using dynamic covalent chemistry allows exploring a wide range of structural components and molecular interactions with a minimal synthetic effort. Here, we describe how non-covalent attractive forces in pseudopeptidic building blocks can succeed in guiding the product distribution in a dynamic library towards topologically more complex compounds that are in principle not expected. The interactions described herein are highly dependent on molecular architecture and media so effective recognition can be altered by just minimal structural or environmental changes. Thus, the chemical and constitutional information contained in the respective building blocks is decoded and expressed through dynamic covalent and non-covalent bonds in the assembling of either a single macrostructure or an ensemble of components with larger structural diversity. The understanding of supramolecular forces responsible for the component assembly in minimalistic systems can help to comprehend more complex biorelated processes such as protein folding or protein-protein interactions
\end{abstract}

\section{Introduction}

Pseudopeptidic compounds are intriguing molecules ${ }^{[1]}$ that have shown very remarkable applications in various fields, such as supramolecular chemistry, ${ }^{[2]}$ biological chemistry ${ }^{[3]}$ and in the preparation of nanomaterials. ${ }^{[4]}$ The combination of abiotic fragments that can confer enzymatic stability with the chemical information that derive from the amino acid functionalities make them especially attractive fragments to study molecular interactions in simplified models with biological significance. ${ }^{[1 \mathrm{~b}]}$ The variety of functionalities present in natural amino acids ${ }^{[5]}$ enables to generate diversity and to explore a plethora of noncovalent contacts. In this context, dynamic constitutional (covalent) chemistry (DCC) ${ }^{[6]}$ provides a valuable tool to study intramolecular and intermolecular interactions. DCC allows generating mixtures of compounds using reversible bond formation which allows the exchange between different subspecies (different building blocks) giving rise to diverse

[a] Dr. M. Lafuente, Dr. I. Alfonso, Dr- J. Solà

Department of Biological Chemistry

Institute for Advanced Chemistry of Catalonia

c/ Jordi Girona 18-26. Barcelona 08034. Spain

E-mail: jordi.sola@iqac.csic.es; Ignacio.alfonso@iqac.csic.es

Supporting information for this article is given via a link at the end of the document. libraries. ${ }^{[7]}$ As the composition of the mixture is determined by the stability of each member and the global energy of the system, the predominance of unexpected compounds or deviation from 'statistical' libraries allows to identify different self-recognition ${ }^{[8]}$ or even autocatalytic processes, being the emergence of selfreplicating systems ${ }^{[9]}$ maybe the most relevant example for its significance in the origin of life. ${ }^{[10]}$ Thanks to the ability of DCC to generate complexity, the multiple potential applications and the advance in analytical tools that now enable dealing with mixtures, the study of dynamic systems is receiving increasing interest. In the last years, we have focused on the study of covalent dynamic libraries based on pseudopeptidic compounds in aqueous media. ${ }^{[1]}$ We found that disulfide chemistry is especially suited for this purpose as we developed conditions that allow us to rapidly form libraries in a wide range of $\mathrm{pHs} .{ }^{[12]}$ In addition, we showed that some of these libraries respond to external stimuli such as the increase of ionic strength or the presence of biomolecules. ${ }^{[11]}$ This methodology had also been employed by different research groups to obtain receptors for relevant biomolecules, ${ }^{[13]}$ but also has been proved a valid tool for the synthesis of topologically complex molecules like catenanes, ${ }^{[14]} \operatorname{knots}^{[8 \mathrm{a}]}$ and even structures that fold like proteins (foldamers). ${ }^{[15]}$

During our investigations we became particularly interested in increasing molecular and topological diversity to generate more complex systems. Commonly, most of the libraries described earlier were based on $C_{2}$ symmetric bifunctional components and, therefore, they combine to assemble relatively complex structures that can always close one or several links forming macrocycles. ${ }^{[8 a}$, 9c, 13-14, 16] Alternatively, although less explored, the combination of building blocks having different geometries and different number of functionalities that form dynamic bonds can give rise to other final structures. ${ }^{[17]}$ We decided to study dynamic libraries that could potentially form structures other than macrocycles in aqueous environments. To this aim, we began to investigate libraries obtained by mixing pseudopeptides with a different number of thiol functional groups, in other words, different 'valence' in the DCC process. In these libraries we could eventually observe self-recognition processes able to drive the library towards the exclusive formation of a single structure. ${ }^{[8 b, c]}$ Later on, this knowledge allowed us to rationally design a dynamic chemical network that reorganizes in the presence of an analyte (cysteine or cystine) liberating a fluorescent compound as readable output. Thus, by knowing the exact nature of the interactions we were able to generate a system that acts as a sensor for a biologically relevant molecule. ${ }^{[18]}$ This example illustrates that understanding the recognition processes that guide virtually complex libraries is very appealing and allows revealing structural and chemical complementarities that can be further used in the design of molecular devices. ${ }^{[19]}$ In this article, we show 
two dissimilar behaviors in the final output of chemical libraries that involve analogous compounds which may only differ in just one methylene unit. In the absence of complementarity, a wide structural diversity of compounds is formed, as expected. However, in the case of a perfect chemical and structural match between a tripodal building block (containing 3 thiol functionalities) and a bipodal moiety (a member that contains two $\mathrm{SH}$ ) an unexpected [2+2] compound emerges selectively from the potential complex mixture as a single product. Our results illustrate how subtle changes in the structure of one compound result in big changes in the final outcome of the whole system. Moreover, the delicate structural and functional information contained in the BBs define the final composition of the library, in the way of either a unique macro-assembly or a complex mixture.

\section{Results and Discussion}

The pseudopeptidic building blocks necessary for the generation of the different libraries under study are accessible by straightforward peptide synthesis protocols in solution (Figure 1). Tripodal compound $\mathbf{1}$ and bipodal unit $\mathbf{3}$ were obtained in two steps from $\mathrm{H}$-Cys(Trt)OtBu and the corresponding trimesic ${ }^{[17 b]}$ and isopthalic ${ }^{[20]}$ acids respectively. Bipodal components 2a-e were obtained in a four step-procedure using the methodology described earlier in our group (Figure 1, see Supporting Information for detailed synthetic procedures). ${ }^{[11 c]}$<smiles>O=C(N[C@@H](CS)C(=O)[O-])c1cc(C(=O)N[C@@H](CS)C(=O)O)cc(C(=O)N[C@@H](CS)C(=O)O)c1</smiles><smiles>[R]CC(NC(=O)CS)C(=O)Nc1cccc(NC(=O)[C@H](C[R])NC(=O)CS)c1</smiles>

Figure 1. Chemical structures of the pseudopeptidic building blocks studied in this article.

Compound distribution of a DCL containing different functional groups. We started by analyzing the products formed in a dynamic library resulting from mixing the tripodal scaffold 1 and different bipodal units with different functionalities and charge (2a-c) in an individual and equimolar manner. The reactions were performed in aqueous media containing $25 \%$ of DMSO at $\mathrm{pH} 6.5$ using a bis-tris buffer $(50 \mathrm{mM}) ; 0.5 \mathrm{mM}$ concentrations for each of the components were used in each experiment. Not surprisingly, the mixtures that contained $\mathbf{1}$ and either $\mathbf{2 a}$ or $\mathbf{2 b}$ revealed the formation of several products with different architectures as can be detected by HPLC-MS analysis. As expected, the negatively charged compound $\mathbf{1}$ efficiently combined with neutral $\mathbf{2 a}$, the major compounds joined two units of $\mathbf{1}$ with either two or three molecules of $2 a\left(\left[\mathbf{1}_{2}-\mathbf{2}_{2}\right],\left[\mathbf{1}_{2}-\mathbf{2}_{3}\right]\right)$. In addition, the corresponding homodimers ([1 $\left.\left.\mathbf{1}_{\mathbf{2}}\right],\left[\mathbf{2} \mathbf{a}_{\mathbf{2}}\right]\right)$ were also detected as minor components (Figure 2A). Interestingly, when $\mathbf{1}$ was combined with a negatively charged bipodal compound $\mathbf{2} \mathbf{b}$ the system was driven towards the formation of the homodimeric species that are presumably more stable than the mixed compounds, which would bring together more negative charges (Figure $2 \mathrm{~B}$ ). However, when the same experiment was performed with the negatively charged 1 and a positively charged bipodal component, 2c, an exclusive single product was formed, corresponding to a heterotetramer [12-2 $\left.\mathbf{c}_{2}\right]$ containing two tripodal units (1) and two bipodal building blocks 2c (Figure 2C). The newly formed species showed a remarkable stability as the library showed no alteration when $2.5 \mathrm{mM}$ of Lcysteine (enough to saturate all the library thiols) was added to the mixture (Figure S19). We reasoned that this stability would be due to the establishment of highly stabilizing forces between the counterparts that would find a perfect fit in charge and space.

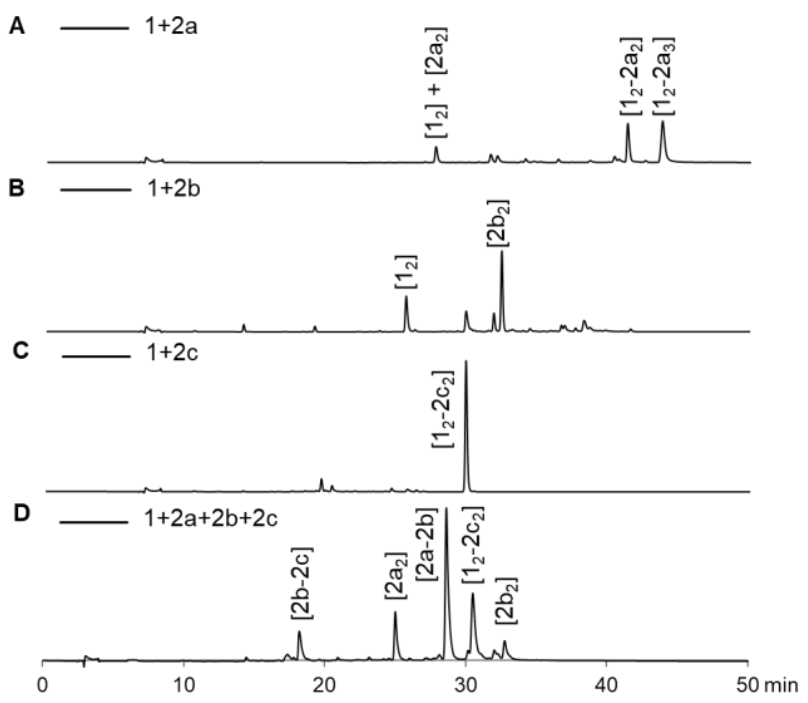

Figure 2. HPLCs traces (UV detection at $254 \mathrm{~nm}$ ) for the DCLs formed by mixing different components. (A): 1 and 2a. (B): 1 and $\mathbf{2 b}$. (C): 1 and 2c. (D) A competition experiment containing $\mathbf{1}, \mathbf{2 a}, \mathbf{2} \mathbf{b}$ and $\mathbf{2 c}$. All reactions contained 0.5 $\mathrm{mM}$ of each component in aqueous solution (25\%DMSO, $\mathrm{pH} 6.5$ ).

This huge stability was further confirmed by other control experiments: increasing amounts of cysteamine (2mercaptoethylamine, CyA) were added in an attempt to disrupt the formation of the tetramer; however, it was necessary to add up to 50 times more cysteamine with respect to the other BBs to start forming other species (Figure S24). Moreover, in a library containing equimolar quantities of 1 and $2 a, 2 b$ and $2 c$ in $a$ competition experiment, all the tripodal 1 was consumed by the formation of $\left[\mathbf{1}_{2}-\mathbf{2}_{2}\right]$ indicating a particular fit for the formation of this compound from $\mathbf{1}$ and $\mathbf{2 c}$ (Figure 2D).

Effect of the reaction media. At this point we reasoned that the divergence in the libraries behavior must be due to the 
establishment of strong non-covalent stabilizing interactions between the functional groups of the amino acid side-chains present in each of the counterparts. To prove the polar nature of these interactions we varied the reaction conditions (polarity and $\mathrm{pH}$ ) and evaluated the outcome of the reaction. As expected, the selectivity towards the formation of the selected tetramer compound was greatly affected by the polarity of the environment: an increase of the ionic strength of the medium led to a diminished predominance of the [2+2] component. The addition of $\mathrm{NaCl}$ prevents the formation of $\left[\mathbf{1}_{2}-2 \mathbf{c}_{2}\right]$ and its area in the HPLC-UV dropped from more than $95 \%$ in the absence of salt to less than $30 \%$ when $1.0 \mathrm{M} \mathrm{NaCl}$ was added to the reaction; the addition of more salt had then a limited effect (Figure 3, blue trace). Nonsurprisingly, the $\mathrm{pH}$ also plays a key role in the nature of the species formed. The optimal $\mathrm{pH}$ for the formation of $\left[\mathbf{1}_{2}-\mathbf{2} \mathrm{c}_{2}\right]$ within the building blocks during the assembly process is around 6.5; at this $\mathrm{pH}$ the amino side-chains are positively charged whereas the carboxylic acid functionalities are in their carboxylate form. Moving away from this value changes the protonation state of either basic or acidic side-chains and, as a result, it is detrimental for self-recognition (Figure 3, red trace). These combined results highlight the nature of polar interactions (salt bridges and hydrogen bonds) that are responsible for the emergence of a single species. Interestingly, the high selectivity towards [12-2 $\left.\mathbf{c}_{\mathbf{2}}\right]$ formation was retained down to $1 \%$ DMSO (Figure S30) while lower concentrations of DMSO resulted in partial precipitation, which precluded a reliable analysis of the composition of the libraries.

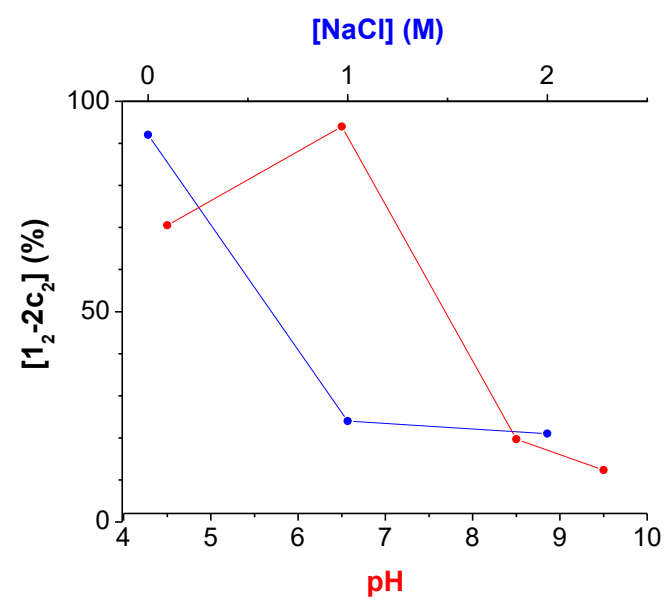

Figure 3. Abundance of $\left[\mathbf{1}_{2}-2 c_{2}\right]$ expressed as percentage of the compound in the HPLC-UV traces (non-corrected by the specific absorbance of each component). Red: effect of $\mathrm{pH}$. Blue: effect of ionic strength

Structural characterization of the predominant species and study of the factors that caused its emergence. The high selectivity observed for the formation of the heterotetramer between $\mathbf{1}$ and $\mathbf{2 c}$ is quite intriguing and, accordingly, we attempted to determine the exact structure and the topology of the self-selected species. For $\left[\mathbf{1}_{\mathbf{2}}-\mathbf{2} \mathbf{c}_{\mathbf{2}}\right]$ there are two possible symmetric (see below) constitutional isomers that can be described as a bis(macrocyclic) structure la (Figure 4A) and a macrobicyclic structure Ib (Figure 4B). In order to determine the connectivity between each counterpart, we synthesized compound $\left[\mathbf{1}_{2}-2 c_{2}\right]$ on a preparative scale and, once isolated, we analyzed its structure by different 1D and 2D NMR experiments in DMSO- $d_{6}$ (Figures S93-S97). The spectra showed a highly symmetric structure in solution $\left(D_{2}\right.$ symmetry) and all the signals could be successfully assigned by the combination of the corresponding NMR experiments (Figure 4D).

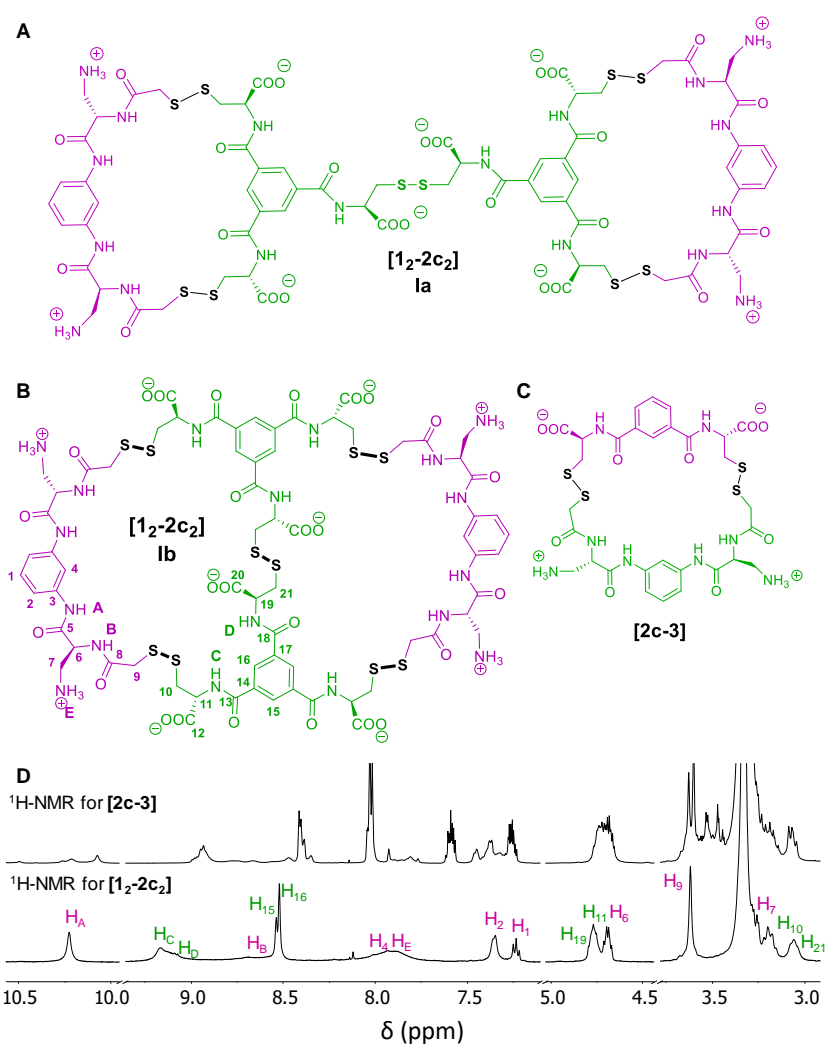

Figure 4. Structure for compound $\left[\mathbf{1}_{\mathbf{2}}-\mathbf{2} \mathbf{c}_{\mathbf{2}}\right]$, la $(A)$ and $\mathbf{l b}(B)$. C) Structure of compound [2c-3], D) Comparative ${ }^{1} \mathrm{NMR}$ spectra $\left(500 \mathrm{MHz}\right.$, DMSO- $d_{6}, 298 \mathrm{~K}$ ) for molecules $\left[\mathbf{1}_{2}-2 c_{2}\right]$ and $[2 c-3]$.

Unfortunately, at least in theory, the two possible isomers could show this $D_{2}$ average symmetry and therefore an unambiguous assignment was not possible at this point. Consequently, we looked for further indications on the nature of the product formed. We reasoned that the comparison of the ${ }^{1} \mathrm{H}-\mathrm{NMR}$ spectrum with that of a simpler but related compound would bring some light into the discussion. To this aim, we analyzed the library formed by reacting $\mathbf{2 c}$ and compound $\mathbf{3}$ which is a structural analogue of tripodal unit 1 but lacking one of the peptidic arms. In this occasion, the simple monomacrocycle [2c-3] arose as the main compound of the mixture, while evidences of larger species were not found (Figure S23); [2c-3] only forms one particular macrocycle with a defined size (Figure $4 \mathrm{C}$ ). In order to compare the NMR spectra of $\left[\mathbf{1}_{\mathbf{2}}-\mathbf{2} \mathrm{c}_{2}\right]$ and [2c-3] the latter was also synthesized on a preparative scale. The ${ }^{1} \mathrm{H}-\mathrm{NMR}$ spectra of $\left[\mathbf{1}^{-}\right.$ 
2c $\mathbf{c}_{2}$ ] and [2c-3] showed significant differences; the ${ }^{1} \mathrm{H}-\mathrm{NMR}$ spectrum of [2c-3] in DMSO- $d_{6}$ presents a much larger number of signals with different intensities suggesting the presence of different conformations of the macrocyclic ring that interconvert slowly in the NMR chemical shift timescale. Moreover, for each set of the ${ }^{1} \mathrm{H}$ NMR signals, the expected $C_{2}$ symmetry was broken in solution (Figure 4D). This observation clearly contrasted with the solution behavior of [1 $\left.\mathbf{1}_{2}-\mathbf{2} \mathrm{c}_{2}\right]$ that showed a full $D_{2}$ symmetry in solution. The putative bis(macrocyclic) heterotetramer la can be described as two [2c-3] macrocycles connected by a cystine bridge, and thus a similar conformational behavior would be expected for la and [2c-3] macrocycles. Considering this evidence and our previous experience with similar macrocyclic disulfides, ${ }^{[21]}$ we concluded that the observed isomer for $\left[\mathbf{1}_{\mathbf{2}}-\mathbf{2} \mathbf{c}_{\mathbf{2}}\right.$ ] corresponded to the macrobicyclic structure $\mathbf{l b}$, where the larger ring size leads to a more flexible structure in solution.

With these experiments, we became confident about the nature of the $\left[\mathbf{1}_{2}-2 \mathbf{c}_{2}\right]$ compound giving a $\mathbf{l b}$ structure and ruling out the bis(macrocyclic) topology la, which necessarily presents the same macrocyclic moiety as [2c-3]. In addition, we performed some further control experiments. MALDI-TOF analysis also confirmed the mass of the [2+2] compound demonstrating the formation of a heterotetramer. Moreover, MALDI-TOF MS/MS experiments were attempted to break the structure through collision-induced dissociation (CID) mode using argon as the collision gas. In these experiments we obtained different fragments of the structure (Figure S101) but we were unable to detect the fragment corresponding to [1-2c] ${ }^{+}$, which would be expected by the symmetric rupture of structure la, or any compound resulting from a one-single bond cleavage. Instead, the loss of either a disulfide unit, $\mathrm{CH}_{2} \mathrm{SS}$ or $\mathrm{CO}_{2}$ were detected. Furthermore, the formation of $\left[\mathbf{1}_{\mathbf{2}}-\mathbf{2} \mathbf{c}_{\mathbf{2}}\right]$ was monitored over time (Figure 5 and Figures S25-S26). At the beginning of the reaction, we observed the immediate formation of a complex mixture that led to the appearance of [1-2c] species (Figure 5). Remarkably, at those initial stages of the reaction, an isomer of the heterotetramer (same $\mathrm{m} / \mathrm{z}$ ) was observed with a different retention time than the final $\left[\mathbf{1}_{\mathbf{2}}-\mathbf{2} \mathbf{c}_{\mathbf{2}}\right]$ compound (see HPLC at 10 min of reaction in Figure 5). We tentatively assigned this peak to the bis-macrocyclic compound la. At three hours the library started to simplify and smoothly evolved to the unique formation of the final macrobicycle la after $24 \mathrm{~h}$, thus taking longer than other libraries previously reported in our group to reach a thermodynamic equilibrium. ${ }^{[8 c]}$ These data suggest that the formation of the main species is fairly complex and, again, this fact supports the formation of $\mathbf{l b}$ in detriment of la, which was transiently formed rather quickly.

A similar experiment was carried out but monitoring the destruction of the $\left[\mathbf{1}_{2}-\mathbf{2 c}_{2}\right]$ compound in the presence of several equivalents of a monothiol (cysteamine, CyA) which can act as a reducing agent of the disulfide linkage and also promote and participate in the dynamic exchange of this bond. Moreover, in the reaction conditions this small amine should be protonated interfering with the stabilizing contacts of the macrobicycle [1 $\mathbf{1}_{2-}$ $2 \mathbf{c}_{2}$ ]. This experiment again showed the remarkable stability of the heterotetramer species. Adding an equimolar amount of this thiol had no effect on the HPLC-traces distribution (Figure 6A).

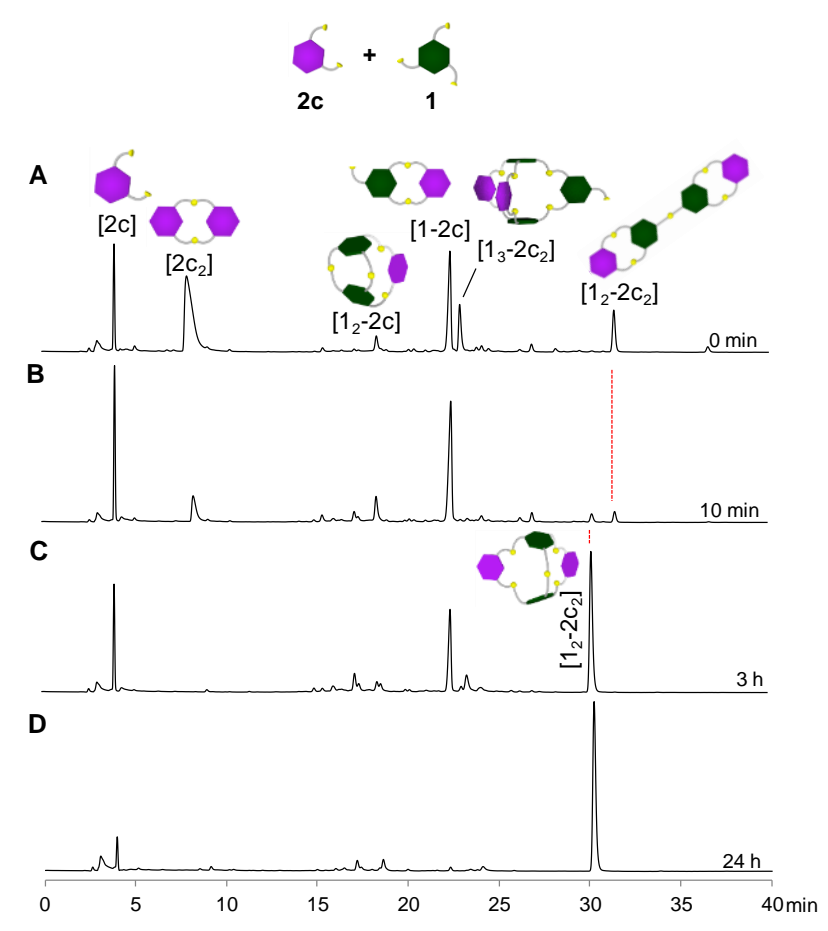

Figure 5. Time-course of the heterotetramer formation: HPLC traces at $t=0$ $\min (A) ; 10$ min $(B) ; 3 h(C)$ and after 24h (D).

It was necessary to add $2.5 \mathrm{mM}$ of CyA (5 equivalents) to start observing the formation of several newly formed species: the bipodal and tripodal species combined with 2 and 3 units of CyA respectively, a heterotrimer containing the three fragments, and a trimer containing two tripodal and one bipodal fragments [12-2c]. The addition of up to $10 \mathrm{mM}$ of CyA increased the abundance of the above mentioned compounds formed by the rearrangement of $\left[\mathbf{1}_{2}-\mathbf{2} \mathbf{c}_{2}\right]$ but with this compound is still present in a notable proportion, which again illustrates its stability.

To gain a deeper knowledge about the structure of the hetero tetramer, we also performed molecular modelling studies using the Macromodel software (Molecular mechanics calculations with the OPLS3 force field in water). Therefore, several systematic Monte Carlo conformational searches were undertaken, each one starting from different initial conformations of the two possible symmetric isomers of the [2+2] structure (la and $\mathbf{l b}$ ). The lowest energy conformers for both isomers are shown in Figure 7. In both cases, the conformations of the systems are ruled by the polar interactions between the charged residues. Thus, in the two minima, the six carboxylate anions are implicated in salt bridges and the structures show a large number of $\mathrm{H}$-bonds (14 in each geometry). These produce quite compact and folded structures. However, the main differences are due to the geometry of the aromatic bis(amide) moieties from the bipodal BBs (highlighted with green C-atoms in Figure 7). 

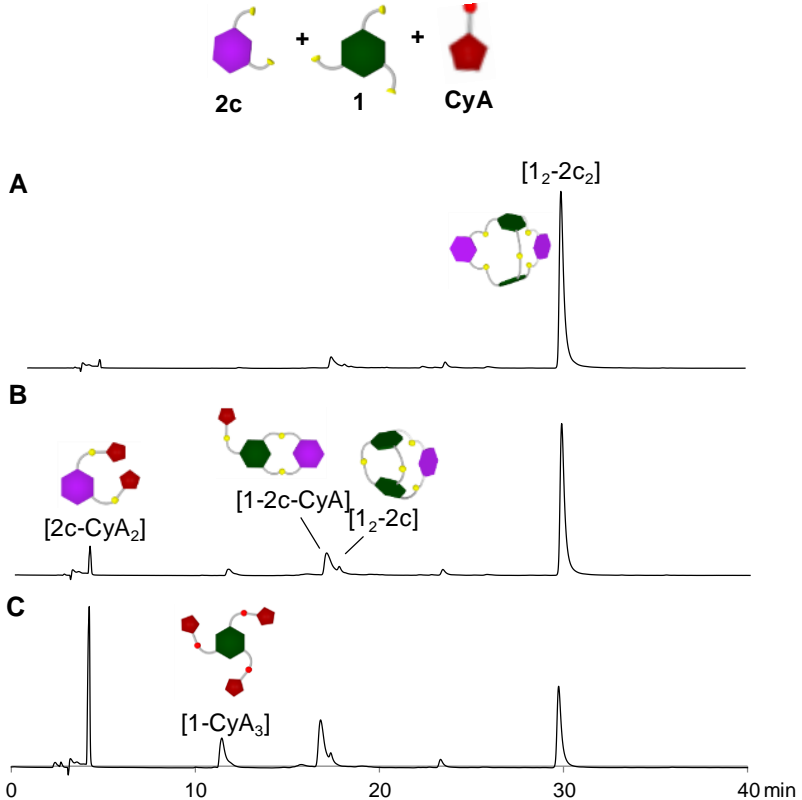

Figure 6. HPLCs traces (UV detection at $254 \mathrm{~nm}$ ) at $24 \mathrm{~h}$ reaction time after the addition of various amounts of cysteamine to a solution containing $0.5 \mathrm{M}$ of [1 $\mathbf{1}_{2}$ 2c $\mathrm{c}_{2}$ (25\% DMSO, pH 6.5). (A) $0.5 \mathrm{mM} \mathrm{CyA;} \mathrm{(B)} 2.5 \mathrm{mM} \mathrm{CyA;} \mathrm{(C)} 10 \mathrm{mM}$ CyA.

In la, the smaller ring size imposes a more strained geometry that precludes the co-planarity of the aromatic bis(amide) fragments, which is reflected in the syn/anti relative disposition of the amide bonds in both moieties. On the contrary, the same fragments in the $\mathbf{l b}$ isomer are co-planar to the aromatic ring and with $\mathbf{a}$ symmetric syn/syn conformation, pointing their $\mathrm{N}-\mathrm{H}$ bonds to the inner part of the macrobicycle ring. This is in agreement with the symmetry observed in the NMR spectra of $\left[\mathbf{1}_{2}-\mathbf{2} \mathbf{c}_{2}\right]$, where a single signal was observed for these four aromatic amide $\mathrm{NH}$ protons. Moreover, the $\mathbf{l b}$ macrobicyclic isomer has a lower energy than the bis-macrocycle la (by $13.6 \mathrm{~kJ} / \mathrm{mol}$ from OPLS3 Force Field). Despite this energy difference could be overestimating the strain effects in a quite flexible structure, the obtained results additionally support the spontaneous formation of the $\mathbf{~} \mathbf{l b}$ isomer over the la counterpart, since the dynamic mixture of disulfides operates under thermodynamic control leading to the most stable situation.

The molecular model for the lb structure also gives some clues about the reasons for the selectivity toward this macrobicycle. The carboxylate anions from the tripodal BBs are tightly $\mathrm{H}$-bound to the amide and ammonium groups of the bipodal BBs. In most of the simulations, these interactions show a chelate-like disposition of the $\mathrm{CH}_{2} \mathrm{NH}_{3}{ }^{+}$side chains of the 2c moiety in a cyclic $\mathrm{H}$-bond geometry (Figure 7 , red dotted ovals). This binding should be highly favored for the short residues, which decrease the conformational freedom of the cationic side chains. We expect that the increase in the flexibility of the cationic residues would disfavor this arrangement, as it will be shown in the next section.
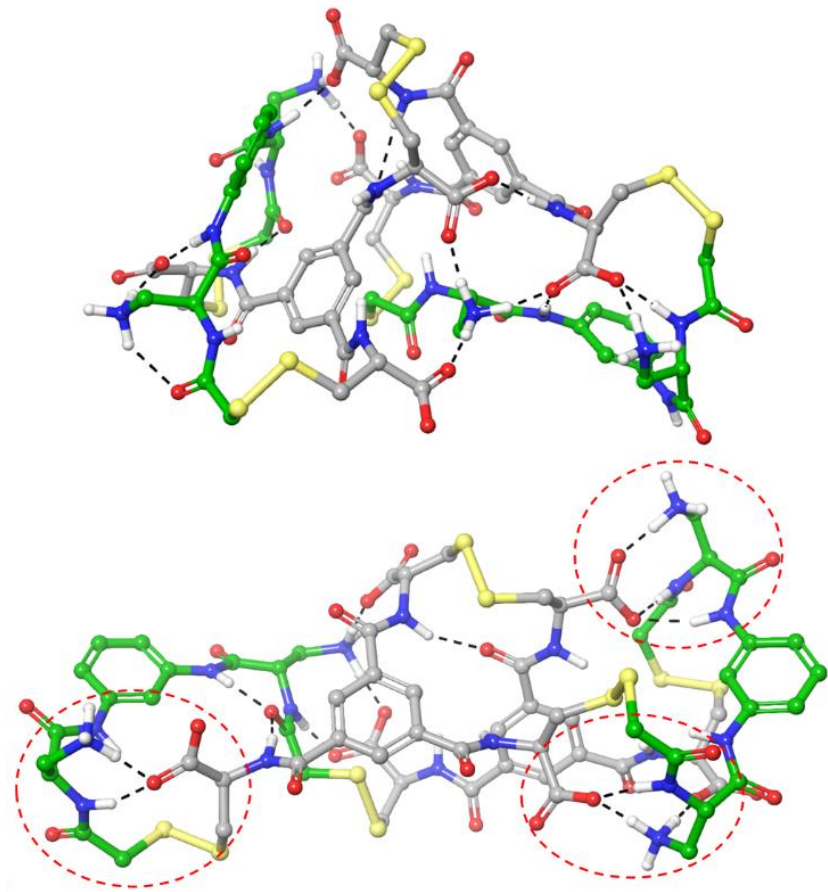

Figure 7. Energy minima for la (up) and lb (down) heterotetramer, obtained after conformational searches (Macromodel, OPLS3 force field in water). For clarity, non-polar hydrogen atoms are not shown, while the carbon atoms from the bipodal BB moiety (2c) are colored in green. The $\mathrm{H}$-bonds are shown with black dashed lines.

Effect of side-chain length: the knowledge acquired in the previous sections allows us to predict the outcome of dynamic libraries composed of very similar building blocks. According to our hypothesis, in a library formed by analogous compounds with different side-chain lengths only the [2+2] adduct of $\mathbf{1}$ and 2c should form whereas the rest of the components would combine to yield other species. To test our assumption several homologous amino compounds with different chain length (2 or 3 methylene units, corresponding to amino acids Dab (2d) and Orn (2e)) were prepared and investigated in dynamic libraries with the trimesic acid-cysteine derivative 1. As expected, when 1 (0.5 mM) was mixed with either compound $\mathbf{2 d}$ or $2 \mathbf{e}(0.5 \mathrm{mM})$ in the usual conditions no predominance towards the [2+2] tetramer was observed for these homologous, positively charged compounds (Figure S20-S21). More strikingly when $1(0.5 \mathrm{mM})$ was reacted with a mixture of $\mathbf{2 c}, \mathbf{2 d}$, and $\mathbf{2 e}(0.5 \mathrm{mM}$ each) in a competition experiment under the usual conditions (aqueous solution at $\mathrm{pH}$ $6.5,25 \% \mathrm{DMSO}$ ) compound $\left[\mathbf{1}_{2}-\mathbf{2} \mathbf{c}_{2}\right]$ was formed in a way that consumed all 1 and $2 \mathrm{c}$ building blocks (Figure $8 \mathrm{~A}$ ). Then, the remaining bipodal components formed the corresponding homo and heterodimers (as revealed by HPLC-MS analysis). Thus, for these structures the formation of $\left[\mathbf{1}_{\mathbf{2}}-\mathbf{2} \mathbf{c}_{\mathbf{2}}\right]$ is exclusive even in the presence of other competing charged species as part of the library. Therefore, the chain length plays a decisive role for the stabilization of a major compound through supramolecular interactions and homologous compounds differing as little as one methylene unit behave in a divergent manner. The delicate 
structural complementarity was further shown by an analogous experiment using bipodal compound $\mathbf{3}$ instead of the tripodal unit 1. In this case, when a mixture containing equimolar quantities of the homologous amino components $\mathbf{2 c}, \mathbf{2} \mathbf{d}$ and $\mathbf{2 e}$ and the bipodal carboxylate $\mathbf{3}$ was tested, no selectivity towards any of the amino derivatives was observed (Figure 8B). Accordingly, compound $\mathbf{3}$ was combined with the different positively charged bipodal units, obtaining as a result a wide range of products in the final distribution of the compounds.

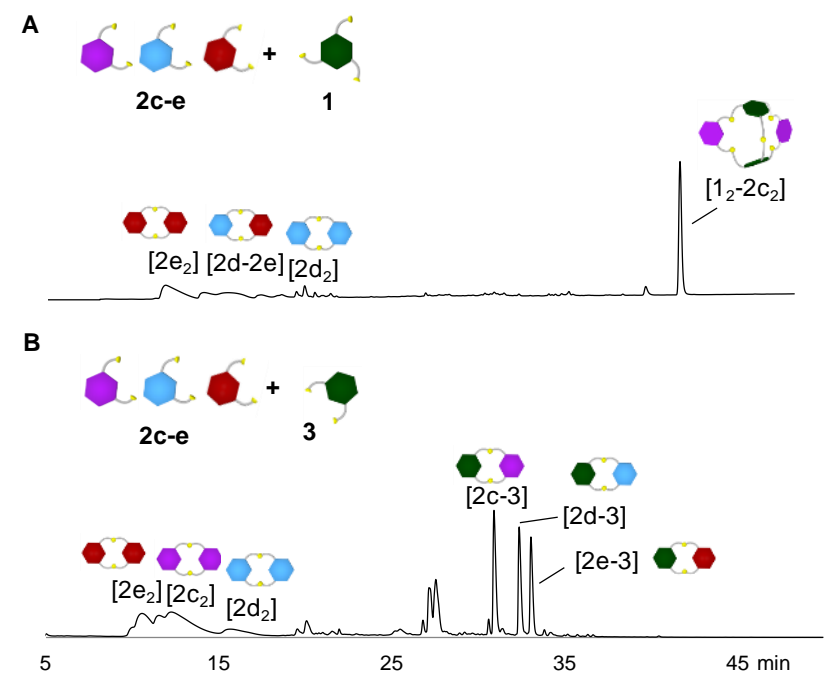

Figure 8. A) DCL formed by 1 and 2 c, d, e $(0.5 \mathrm{mM}$ each) in aqueous solution (25\% DMSO, pH 6.5), B) DCL formed by 3 and $2 \mathrm{c}-\mathrm{e}(0.5 \mathrm{mM}$ each) in aqueous solution (25\% DMSO, pH 6.5).

Once again, these results indicate the delicate structural complementarity necessary for the emergence of self-recognition processes. In particular, this experiment demonstrates the need of the third arm to selectively form $\left[\mathbf{1}_{\mathbf{2}}-\mathbf{2} \mathbf{c}_{2}\right]$ and it is a further evidence for the $\mathbf{l b}$ isomer as it forms a bigger macrocycle which brings the charges with opposite sign in close proximity. This disposition would explain the dissimilar behavior for the other bipodal units where a single chain extension prevents establishing the adequate stabilizing interactions. These experimental results are in agreement with the computational calculations described in the previous section; the conformational freedom of the larger residues would preclude adopting the closed conformation necessary to establish these polar interactions.

\section{Conclusions}

We have shown the intricate relationships that govern the product distributions in dynamic libraries of pseudopeptides. While an expected, statistical, product distribution consisting in homo and heterodimers is found for most of the libraries, in some cases the equilibrium is driven towards the formation of an unexpected heterotetramer. In order to form this discrete macrobicyclic structure, stabilizing interactions must come in place but the effect of these non-covalent interactions is sufficient to change the size of the macrocycles formed from 26 to 42 members. The polar nature of these contacts is demonstrated by the reduction of the major product when the reactions were performed in different $\mathrm{pH}$ or in a higher ionic strength media. The discrimination was shown to be also very sensitive to the size of the building blocks being just an increment of a methylene unit detrimental for efficient formation. In addition, the removal of a covalent link between two of the components also prevents the formation of the tetrameric species. The formation of the major compound is therefore, directed by the nature of all counterparts. The stability of the newly formed compound has been shown by different competition experiments with charged monothiols and by MS-MS spectrometry. The structural importance of intramolecular interactions in aqueous media is shown in many biological processes but is especially relevant in the folding of proteins where the system adopts a tridimensional structure that is not only maintained by covalent bonds but by other non-covalent interactions. Accordingly, the understanding of simpler systems can help to explain the behavior of such complex structures. In this context, Dynamic Covalent Chemistry constitutes a powerful tool to discover non-trivial recognition processes. Knowing the rules that guide self-assembly will allow us to design more complex systems and predict their behavior, opening the door to the synthesis of molecular devices with a tailored function.

\section{Experimental Section}

Syntheses of compounds $\mathbf{1},{ }^{[17 b]} \mathbf{3}^{[20]}$ and $\mathbf{2 a}, \mathbf{2} \mathbf{b}, \mathbf{2} \mathbf{e}^{[11 c]}$ have been previously reported. Synthetic details for the preparation of $\mathbf{2 c}, \mathbf{2 d}$ can be found in the ESI.

Reversed-Phase High-Performance Liquid Chromatography (RP-HPLC) analyses were performed on a Hewlett Packard Series 1100 (UV detector $1315 \mathrm{~A})$ modular system using a reversed-phase kromaphase $\mathrm{C}_{18}(25 \mathrm{x}$ $0.46 \mathrm{~cm}, 5 \mu \mathrm{m})$ column. $\left(\mathrm{CH}_{3} \mathrm{CN}+20 \mathrm{mM} \mathrm{HCOOH}\right.$ and $\mathrm{H}_{2} \mathrm{O}+20 \mathrm{mM}$ $\mathrm{HCOOH}$ ) mixtures at $1 \mathrm{~mL} / \mathrm{min}$ were used as mobile phase and the monitoring wavelength was set at $254 \mathrm{~nm}$.

$\mathrm{pH}$ measurements were performed at room temperature on a Crison GLP21 pH-meter with the electrodes Crison 50 14T ( $\geq 10 \mathrm{~mL}$ samples) and PHR-146 Micro (<10 mL samples).

High Resolution Mass Spectrometry (HRMS) analyses were carried out at the IQAC Mass Spectrometry Facility, using a UPLC-ESI-TOF equipment: [Acquity UPLC® BEH $\mathrm{C}_{18} 1.7 \mathrm{~mm}, 2.1 \times 100 \mathrm{~mm}$, LCT Premier Xe, Waters] $\left(\mathrm{CH}_{3} \mathrm{CN}+20 \mathrm{mM} \mathrm{HCOOH}\right.$ and $\left.\mathrm{H}_{2} \mathrm{O}+20 \mathrm{mM} \mathrm{HCOOH}\right)$ mixtures at 0.3 $\mathrm{mL} / \mathrm{min}$ were used as mobile phase.

General procedure for the preparation and HPLC analysis of the DCLs. A 66.7 mM Bis-Tris methane buffer was prepared by dissolving 1.39 $\mathrm{g}$ of the free amine in $100 \mathrm{~mL}$ of milli-Q water and adjusting the $\mathrm{pH}$ of the solution to 6.5 by the addition of $\mathrm{HCl}(\mathrm{aq})$. The reaction mixtures were prepared by dilution of individual stocks of the building blocks (BBs) 1, 2ae and $\mathbf{3}$ in DMSO. The concentration of the stocks of free thiols was adjusted by a calibration using Ellman's reagent (see Sup. Info. for more details). Unless otherwise specified, the DCLs were prepared at $0.5 \mathrm{mM}$ of the BBs in a $50 \mathrm{mM} \mathrm{BIS-Tris} \mathrm{methane} \mathrm{buffer}(\mathrm{pH} \mathrm{6.5)}$ with $25 \%$ DMSO. The mixtures were analyzed by means of HPLC or UPLC-MS at different 
reaction times. Complete oxidation is achieved after $24 \mathrm{~h}$. The reactions were then analyzed by preparing HPLC samples adding $45 \mu \mathrm{L}$ of the corresponding reaction mixture to $75 \mu \mathrm{L}$ of a solution of $89 \% \mathrm{H}_{2} \mathrm{O}, 10 \%$ $\mathrm{CH}_{3} \mathrm{CN}$ and $1 \%$ TFA. Eluent used: 2 min at $10 \% \mathrm{CH}_{3} \mathrm{CN}$ in $\mathrm{H}_{2} \mathrm{O}$, then linear gradient from $10 \%$ to $50 \% \mathrm{CH}_{3} \mathrm{CN}$ over $58 \mathrm{~min}$.

\section{Acknowledgements}

We thank Dr. Yolanda Pérez and Dr. Carme Quero for their valuable assistance with NMR and MALDI-TOF MS experiments, respectively. Financial Support from MINECO/FEDER (CTQ2015-70117-R and BES-2013-063128), AGAUR (2017 SGR 208) and European Union (COST CM1304) are gratefully acknowledged.

Keywords: dynamic covalent chemistry • self-assembly • noncovalent interactions $\cdot$ peptidomimetics $\bullet$ topology

[1] a) Y.-D. Wu, S. Gellman, Acc. Chem. Res. 2008, 41, 1231-1232; b) I. Alfonso, Chem. Commun. 2016, 52, 239-250.

[2] a) E. Faggi, A. Moure, M. Bolte, C. Vicent, S. V. Luis, I. Alfonso, J. Org Chem. 2014, 79, 4590-4601; b) S. V. Luis, I. Alfonso, Acc. Chem. Res. 2014, 47, 112-124; c) E. Faggi, C. Vicent, S. V. Luis, I. Alfonso, Org Biomol. Chem. 2015, 13, 11721-11731; d) E. Faggi, Y. Pérez, S. V. Luis, I. Alfonso, Chem. Commun. 2016, 52, 8142-8145.

[3] a) P. Kokkonen, M. Rahnasto-Rilla, P. H. Kiviranta, T. Huhtiniemi, T. Laitinen, A. Poso, E. Jarho, M. Lahtela-Kakkonen, ACS Med. Chem. Lett. 2012, 3, 969-974; b) E. Zervoudi, E. Saridakis, J. R. Birtley, S. S. Seregin, E. Reeves, P. Kokkala, Y. A. Aldhamen, A. Amalfitano, I. M. Mavridis, E. James, D. Georgiadis, E. Stratikos, Proc. Natl. Acad. Sci. 2013, 110, 19890-19895; c) N. Micale, K. Scarbaci, V. Troiano, R. Ettari, S. Grasso, M. Zappalà, Med. Res. Rev. 2014, 34, 1001-1069.

[4] a) M. Reches, E. Gazit, Science 2003, 300, 625-627; b) L. Ziserman, H. Y. Lee, S. R. Raghavan, A. Mor, D. Danino, J, Am. Chem. Soc. 2011 133, 2511-2517; c) V. Haridas, M. B. Bijesh, A. Chandra, S. Sharma, A Shandilya, Chem. Commun. 2014, 50, 13797-13800.

[5] G. C. Barrett, D. T. Elmore, Amino Acids and Peptides, Cambridge University Press, Cambridge, 1998

[6] a) J.-M. Lehn, Angew. Chem. Int. Ed. Engl. 1990, 29, 1304-1319; Angew. Chem. 1990, 102, 1347-1362; b) J.-M. Lehn, Chem. Soc. Rev. 2007, 36, 151-160.

[7] P. T. Corbett, J. Leclaire, L. Vial, K. R. West, J.-L. Wietor, J. K. M. Sanders, S. Otto, Chem. Rev. 2006, 106, 3652-3711.

[8] a) N. Ponnuswamy, F. B. L. Cougnon, J. M. Clough, G. D. Pantoş and J. K. M. Sanders, Science 2012, 338, 783-785; b) J. Solà, M. Lafuente, J. Atcher, I. Alfonso, Chem. Commun. 2014, 50, 4564-4566; c) M. Lafuente, J. Atcher, J. Sola, I. Alfonso, Chem. Eur. J. 2015, 21, 1700217009; d) M. Kołodziejski, A. R. Stefankiewicz, J. M. Lehn, Chem. Sci. 2019,10, 1836-1843.
[9] a) J. W. Sadownik, D. Philp, Angew. Chem. Int. Ed. 2008, 47, 99659970; Angew. Chem. 2008, 120, 10113-10118. b) R. Nguyen, L. Allouche, E. Buhler, N. Giuseppone, Angew. Chem. Int. Ed. 2009, 48, 1093-1096; Angew. Chem. 2009, 121, 1113-1116. c) J. M. A. Carnall, C A. Waudby, A. M. Belenguer, M. C. A. Stuart, J. J.-P. Peyralans, S. Otto, Science 2010, 327, 1502-1506; d) M. Colomb-Delsuc, E. Mattia, J. W. Sadownik, S. Otto, Nat. Commun. 2015, 6, 7427; e) J. W. Sadownik, E. Mattia, P. Nowak, S. Otto, Nat. Chem. 2016, 8, 264; f) C. Chen, J. Tan M. C. Hsieh, T. Pan, J. T. Goodwin, A. K. Mehta, M. A. Grover, D. G. Lynn, Nat. Chem. 2017, 9, 799-804.

[10] a) T. Gánti, The Principles of Life, Oxford University Press, Oxford, 2003; b) K. Ruiz-Mirazo, C. Briones, A. de la Escosura, Chem. Rev. 2014, 114 285-366.

[11] a) J. Atcher, A. Moure, I. Alfonso, Chem. Commun. 2013, 49, 487-489 b) J. Atcher, A. Moure, J. Bujons, I. Alfonso, Chem. Eur. J. 2015, 21 6869-6878; c) J. Atcher, J. Sola, I. Alfonso, Org. Biomol. Chem. 2017, 15, 213-219; d) A. M. Valdivielso, F. Puig-Castellvi, J. Atcher, J. Sola, R. Tauler, I. Alfonso, Chem. Eur. J. 2017, 23, 10789-10799.

[12] J. Atcher, I. Alfonso, RSC Adv. 2013, 3, 25605-25608.

[13] a) S. Otto, S. Kubik, J. Am. Chem. Soc. 2003, 125, 7804-7805; b) M. Rauschenberg, S. Bomke, U. Karst, B. J. Ravoo, Angew. Chem. Int. Ed. 2010, 49, 7340-7345.

[14] a) F. B. L. Cougnon, N. Ponnuswamy, N. A. Jenkins, G. D. Pantos, J. K. M. Sanders, J. Am. Chem. Soc. 2012, 134, 19129-19135; b) R. T. S Lam, A. Belenguer, S. L. Roberts, C. Naumann, T. Jarrosson, S. Otto, J. K. M. Sanders, Science 2005, 308, 667-669; c) H. Y. Au-Yeung, G. D. Pantos, J. K. Sanders, Proc. Natl. Acad. Sci. 2009, 106, 10466-10470.

[15] a) C. Tsiamantas, X. de Hatten, C. Douat, B. Kauffmann, V. Maurizot, H. Ihara, M. Takafuji, N. Metzler-Nolte, I. Huc, Angew. Chem. Int. Ed. 2016 55, 6848-6852; Angew. Chem. 2016, 128, 6962-6966; b) B. Liu, C. G. Pappas, E. Zangrando, N. Demitri, P. J. Chmielewski, S. Otto, J. Am. Chem. Soc. 2019 DOI: 10.1021/jacs.8b11698.

[16] S. Otto, R. L. E. Furlan, J. K. M. Sanders, Science 2002, 297, 590-593.

[17] a) A. M. Whitney, S. Ladame, S. Balasubramanian, Angew. Chem. Int. Ed. 2004, 43, 1143-1146; Angew. Chem. 2004, 116, 1163-1166; b) K. R. West, K. D. Bake, S. Otto, Org. Lett. 2005, 7, 2615-2618; c) Y. Krishnan-Ghosh, A. M. Whitney, S. Balasubramanian, Chem. Commun. 2005, 3068-3070; d) A. Bugaut, K. Jantos, J.-L. Wietor, R. Rodriguez, J. K. M. Sanders, S. Balasubramanian, Angew. Chem. Int. Ed. 2008, 47, 2677-2680; Angew. Chem. 2008, 120, 2717-2720; e) W. Drożdż, C. Bouillon, C. Kotras, S. Richeter, M. Barboiu, S. Clément, A. R. Stefankiewicz, S. Ulrich, Chem. Eur. J. 2017, 23, 18010-18018.

[18] M. Lafuente, J. Solà, I. Alfonso, Angew. Chem. Int. Ed. 2018, 57, 8421 8424; Angew.Chem. 2018, 130, 8557-8560.

[19] S. Kassem, T. van Leeuwen, A. S. Lubbe, M. R. Wilson, B. L. Feringa, D. A. Leigh, Chem. Soc. Rev. 2017, 46, 2592-2621.

[20] T. M. Postma, W. R. J. D. Galloway, F. B. L. Cougnon, G. D. Pantos, J. E. Stokes, D. R. Spring, Synlett 24, 765-769.

[21] J. Atcher, J. Bujons, I. Alfonso, Chem. Commun. 2017, 53, 4274-4277. 
Entry for the Table of Contents (Please choose one layout)

\section{FULL PAPER}

Perfect structural and chemical match can lead to compound selection in dynamic libraries even in front of very related compounds

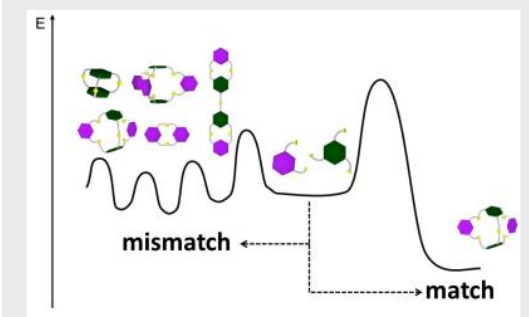

María Lafuente, Ignacio Alfonso*, Jordi Solà*

Page No. - Page No.

Structurally selective assembly of a specific macrobicycle from a dynamic library of pseudopeptidic disulfides. 